\title{
Self-Consistent Field Theory of Ionization Collisions
}

\author{
Essaid Zerrad \\ Department of Physics and Engineering, Delaware State University, Dover, DE, USA \\ Email: ezerrad@desu.edu
}

How to cite this paper: Zerrad, E. (2017) Self-Consistent Field Theory of Ionization Collisions. Journal of Modern Physics, 8 , 1521-1536.

https://doi.org/10.4236/jmp.2017.89091

Received: June 21, 2017

Accepted: August 6, 2017

Published: August 9, 2017

Copyright (C) 2017 by author and Scientific Research Publishing Inc. This work is licensed under the Creative Commons Attribution International License (CC BY 4.0).

http://creativecommons.org/licenses/by/4.0/

\begin{abstract}
A self-consistent field (SCF) theory for treating scattering systems was formulated previously and is extended here to the ionization process, in which the continuum orbitals are made square integrable by an amputation procedure. The method is applied to the electron-hydrogen scattering system in the zero angular momentum coupling model, and the differential cross section is compared with the recent results obtained by several other approaches. It is shown that the amputated continuum functions provide an effective projection of the scattering equation. SCF continuum functions generated in the present formalism are used to analyze the effective charge approximation.
\end{abstract}

\section{Keywords}

Ionization, SCF, $\mathrm{HF}$

\section{Introduction}

For many years there has been a great deal of experimental and theoretical work devoted to the study of electron impact ionization processes and its inverse, the three body recombination. Such processes are of interest in many fields, such as astrophysics, plasma physics, short-wave laser development, fusion research and medical physics. The theoretical description of this process is difficult because the system involves at least two particles in the continua, which interact via long range Coulomb potentials. A self-consistent field (SCF) treatment is even more problematic, due to the non-square integrability of the continuum wave functions. Recently, much theoretical analyses focused on these difficulties have been made, both formally and numerically, and various models have been treated by accurate numerical or variational calculations [1]-[9]. Despite recent theoretical advances [10] [11] the ionization problem still remains as one of the outstanding subjects of theoretical interest. 
In view of the successful application of the Hartree-Fock approach to manyparticle bound state problems in the past, we have attempted a similar treatment for the scattering systems. The conventional theories which have been used in the past for the bound state problems [12] [13] [14] [15] are not quite applicable for scattering and ionization problems, mainly because there are one or more continuum wave functions involved in the description of the final states. Evidently, the continuum functions are not square-integrable i.e. not L2, and this fact complicates the mathematical as well as the computational aspect of the theory. In order to remove this fundamental difficulty, we have developed previously a new procedure [16] [17] that introduces an amputation of the scattering function at large distance, making it square-integrable. Note that, except for the phase shift, the tail of the continuum function carries minimal dynamic information. The use of the amputated functions thus allowed the development of a self-consistent procedure for the evaluation of the scattering amplitude in a systematic way. It is termed a generalized Hartree-Fock theory (GHF). The theory was tested for its validity and applicability [18] [19] as it was successfully applied to the positron-hydrogen and electron-hydrogen scattering systems, and further extended to the positron-helium and electron-helium scattering systems, where the exact target functions are not known. In fact both the scattering and target functions are simultaneously determined self-consistently by the theory.

In the present paper, we extend the GHF theory to the ionization problem, where at least two continuum functions are to be made square-integrable (L2) by the amputation procedure. As the GHF theory focuses on properly treating the continuum function, it is eminently suited for the ionization problem. As preliminary to a full treatment of many physically interesting cases, we study in this report a simple electron-hydrogen scattering in the zero angular momentum approximation. Although the angular momentum is uncoupled, this model problem still contains much of the difficulties of a three-body Coulomb problem, and it also serves as a non-trivial test of the new approach to the ionization problem. In Section 2, the GHF theory for the elastic scattering is summarized and extended to the ionization process. In Section 3, we present the details of ionization of hydrogen by electron impact. Our results are summarized in Section 4. In Section 5, we completely analyze the GHF wave functions and critically examine the effective charge approximation.

\section{Generalized Hartee-Fock Approch}

The GHF for the scattering systems is summarized below, and then extended to the ionization problem. The theory is developed closely paralleling the SCF approach for the bound state case, and the differences in the scattering case are emphasized.

\subsection{Theory}

We summarize the salient properties of the GHF procedure for the simple elastic 
scattering. First, for an N-electron bound system consisting of a valence electron and a neutral atomic core of $N-1$ electrons, the core state configuration assumes in its lowest order approximation a simple anti-symmetrized product of single-particle orbitals, all of which are L2. Thus, in a slightly more convenient form for our purpose below, we have

$$
\Psi \cong \Psi_{H F}=A \prod \varphi_{i}=A \Psi_{-i} \varphi_{i}, \quad i=1,2, \cdots, N
$$

with $\left\langle\varphi_{i} \mid \varphi_{i}\right\rangle=\delta_{i j}$, for each orbital $\varphi_{i}$, and where $\Psi_{-i}$ are the $(N-1)$ electron clusters which may be assumed to be fully antisymmetrized within the clusters. A is the anti-symmetrization operator. The nonlinear set of coupled equations for the individual orbitals $\varphi_{i}$ is then obtained by the projection, as

$$
\left\langle\Psi_{-i}\left|H-E_{t}\right| \Psi_{H F}\right\rangle_{-i}=0, i=1,2, \cdots, N
$$

where \langle\rangle$_{-i}=\int \prod_{i \pm i} \mathrm{~d} \boldsymbol{r}_{i}$. Evidently, all the integrals involved are finite.

For the scattering problem with one or more open channels, $\varphi_{k}$ represents the continuum orbitals. The total wave function then behaves asymptotically as

$$
\Psi \rightarrow \Psi_{t}=A \Psi_{-k} \varphi_{k}, \text { with } \varphi_{k} \rightarrow S_{k}+K_{k} C_{k}
$$

where $\Psi_{-k}$ describes $N-1$ electron target state without the $k$-th (continuum) particle, and $S_{k}$ and $C_{k}$ are the free sine and cosine waves asymptotically. In the case of single channel scattering, the reactance matrix $K$ assumes a simple function $\tan \delta$, where $\delta$ is the scattering phase shift. The normalizability condition on the single-electron orbitals breaks down for the continuum function $\varphi_{k}$, i.e. $\int \mathrm{d} \boldsymbol{r}\left|\varphi_{k}\right|^{2}=\infty$.

To resolve this non-L2 difficulty, we define the amputated wave functions (AWF) $\mathrm{X}$ as

$$
X_{k}=D_{k} \varphi_{k}, \text { where } D_{k} \equiv E_{k}-T_{k},(\mathrm{AWF})
$$

In Equation (2.4), $E_{k}$ is the scattering energy and $T_{k}$ is the kinetic energy operator for the k-th continuum particle. $D_{k}$ cuts off the tail of $\varphi_{k}$, and as a result the AWF X's are now square-integrable. The crucial point to note here is that the amputation does not sacrifice any vital information, since the tail of a continuum function carries very little dynamical information; it mainly contains the phase shift and the wave number for that channel. Therefore, no essential physics is lost by the amputation, but we have gained the L2 property. Furthermore, we replace the strong asymptotic condition (2.3) and the exact $\Psi_{-k}$ by a weak asymptotic condition (WAC)

$$
\left\langle\Psi_{-k t}\left|H_{-k}-E_{-k t}\right| \Psi_{-k t}\right\rangle_{-k}=0,(\mathrm{WAC})
$$

where $-k=(1,2,3, \cdots, k-1, k+1, \cdots, N)$ and where $E_{-k t}$ is the approximate (HF) cluster energy associated the approximate trial function $\Psi_{-k t}$. This is an important relaxation of the original boundary condition; Equation (2.5) not only sets a weak accuracy requirement on the cluster function $\Psi_{-k t}$, which will eventually be improved with the addition of more configurations to the solution, but also allows a SCF determination of both the target and scattering orbitals. 
The generalized SCF equations for scattering are given by the projections, as

$$
\left\langle\Psi_{-k t}\left|H-E_{k}\right| \Psi_{G H F}\right\rangle_{-k}=0,
$$

where $\Psi_{t} \cong \Psi_{G H F}$ is of the form (2.3). Furthermore, for the $\mathrm{j}$-th bound orbitals,

$$
\left\langle\Psi_{-k-j t} X_{k}\left|H-E_{t}\right| \Psi_{G H F}\right\rangle_{-j}=0, j \neq k,(\mathrm{WAC})
$$

where $-k$ - $j$ denotes the $N$-2 particles that exclude both the $\mathrm{k}$-th and $\mathrm{j}$-th particles. This is the desired set of the generalized Hartree-Fock (GHF) equations. Equations (2.6) can be reduced to compact forms

$$
\begin{gathered}
{\left[T_{k}+U_{k}-E_{k}\right] \varphi_{k}=-Y_{k},} \\
{\left[T_{i}+U_{i}-E_{d t i}\right] \varphi_{-i}=-Y_{i}, \quad i \neq k,}
\end{gathered}
$$

where $U_{k}$ and $U_{i}$ are the effective SCF potentials seen by the continuum and bound state functions, respectively. The Y's are the exchange terms involving integrals of $V_{i i}$, and $E_{d t i}$ are the effective energies for the bound orbitals $\varphi_{i}$ for electron $\mathrm{i}$ in the potential $U_{i}$. Because of the non-L2 difficulty, $U_{i}$ with $i \neq k$ for the target orbitals have never been derived previously within the SCF approach. Evidently, Equation (2.7b) is a well-defined equation for the bound state orbitals distorted by the continuum orbital. Also $U$ are totally new result which displays for the first time the SCF potential for the target system during a collision. As a result, the SCF target functions become mildly energy dependent.

As usual, the multi-configuration mixing is realized by writing the total wave functions as $\Psi_{t} \cong A \Psi_{-k} \psi_{k}+\sum \Psi_{n}$, where the last sum is over the closed (or some open) channels in the case of single (or multiple) channel scattering. This generalization will be considered in future reports.

In the previous reports we showed that the GHF theory, with two key ansatze of WAC and AWF, is effective in treating the many-body scattering problem. We have explicitly demonstrated [18] [19] that the WAC converges to the strong (exact) asymptotic condition as the total wave function is improved via multiconfiguration mixing.

\subsection{Ionization by Electron Impact}

The GHF for scattering summarized above can immediately be adapted to the ionization problem of interest here in a natural way, because the amputation can now be carried out on the two continuum functions. The final state wave function is written as

$$
\Psi=A \Psi_{-k-p} \varphi_{k} \varphi_{p}
$$

where $\Psi_{-k-p}$ is the residual target function of $\mathrm{N}-2$ electrons and $\varphi_{k}, \varphi_{p}$ are the continuum functions. The amputation is then carried out simply as

$$
X_{k}=D_{k} \varphi_{k} \text { and } X_{p}=D_{p} \varphi_{p}
$$

where $D_{k}$ and $D_{p}$ may be different, not only in the constant kinetic energies, but also possibly due to the presence of long-range interaction. In some cases, the 
Coulomb potentials may not be completely screened for the residual ions. This point becomes clearer in the discussion of specified systems (See Section 4).

\section{Ionization of Hydrogen by Electron Impact}

We consider the non-relativistic electron scattering by atomic hydrogen scattering with total orbital angular momentum $L=0$ and total angular spin $S=0$, 1. In the center of mass frame the non-relativistic Schrödinger equation for the interaction of electrons with a hydrogen atom is

$$
\left[-\frac{\hbar^{2}}{2 m}\left(\nabla_{1}^{2}+\nabla_{2}^{2}\right)-\frac{e^{2}}{r_{1}}+\frac{e^{2}}{r_{12}}-\frac{e^{2}}{r_{2}}-E_{t}\right] \Psi\left(\boldsymbol{r}_{1}, \boldsymbol{r}_{2}\right)=0,
$$

where $\boldsymbol{r}_{1}$ and $\boldsymbol{r}_{2}$ are the coordinates of the atomic electron and the incident particle respectively, relative to the infinitely heavy proton considered to be at rest, $E_{t}$ is the total energy of the system, and $\frac{1}{r_{12}}=\frac{1}{\left|\boldsymbol{r}_{1}-\boldsymbol{r}_{2}\right|}$.

In the present formalism, the generalized Hartree-Fock (GHF) approach is chosen in its lowest approximation to be of the form

$$
r_{1} r_{2} \Psi\left(\boldsymbol{r}_{1}, \boldsymbol{r}_{2}\right) \cong u_{1}^{l=0}\left(r_{1}\right) u_{2}^{l=0}\left(r_{2}\right) \pm u_{1}^{l=0}\left(r_{2}\right) u_{2}^{l=0}\left(r_{1}\right),
$$

for the singlet $(+)$ and triplet $(-)$ cases respectively. This form automatically defines our model, i.e. $\frac{1}{r_{12}} \rightarrow \frac{1}{r_{>}}$. Note that its converse is not valid, i.e. the model does not necessarily lead to (3.2). In the following the explicit reference to $l_{1}=I_{2}$ $=0$ is omitted to simplify notation. The corresponding amputated wave functions are defined as

$$
X_{i}\left(r_{i}\right)=\left(\frac{\mathrm{d}^{2}}{\mathrm{~d} r_{i}^{2}}+k_{i}^{2}\right) u_{i}\left(r_{i}\right) \text { for } i=1,2,
$$

where the operator $\frac{\mathrm{d}^{2}}{\mathrm{~d} r_{i}^{2}}+k_{i}^{2}$ cuts off the tail of the scattering function $u_{i}$. As a result, the AWF $X$ s are now square integrable as they decay like ordinary bound state wave functions.

The generalized SCF equations are derived by projecting out Equation (3.1) with $\left\langle X_{i}\left(\boldsymbol{r}_{i}\right)\right|$ and integrating over the coordinate $\boldsymbol{r}_{i}$. These equations can be written as

$$
\left(\frac{\mathrm{d}^{2}}{\mathrm{~d} r^{2}}-V_{i}(r)+k_{i}^{2}\right) u_{i}(r)= \pm \int \mathrm{d} r^{\prime} y_{i}\left(r, r^{\prime}\right) u_{i}\left(r^{\prime}\right) \equiv \pm Y_{i},
$$

where $V_{i}(r)$ is the electron-electron interaction potential and given by

$$
V_{i}(r)=-\frac{2}{r}+\frac{\int \mathrm{d} r^{\prime} X_{j}\left(r^{\prime}\right) \frac{2}{r_{>}} u_{j}\left(r^{\prime}\right)}{\int \mathrm{d} r^{\prime} X_{j}\left(r^{\prime}\right) u_{j}\left(r^{\prime}\right)} .
$$

The non-local potential $y_{i}$ describes the electron exchange in which the 2 electrons interchange roles and is defined as 


$$
y_{i}\left(r, r^{\prime}\right)=\frac{X_{j}\left(r^{\prime}\right)\left(\frac{\mathrm{d}^{2}}{\mathrm{~d} r^{2}}+\frac{2}{r}+k_{j}^{2}\right) u_{j}(r)-X_{j}\left(r^{\prime}\right) \frac{2}{r_{>}} u_{j}(r)}{\int \mathrm{d} r^{\prime} X_{j}\left(r^{\prime}\right) u_{j}\left(r^{\prime}\right)}
$$

The $V_{i}^{\prime}$ s are the direct SCF potentials for the $u_{i}^{\prime}$ s, which have never been possible to derive for the continuum electrons.

The transition amplitude $T_{f i}$ is given by

$$
T_{f i}=\left\langle\Psi_{f}\left|H-E_{t}\right| \Psi_{i}^{0}\right\rangle,
$$

$\Psi_{f}$ and $\Psi_{i}^{0}$ represent the wave functions describing the whole system in its fully interacting final state and non-interacting initial state, respectively. The fully differential cross section is given by

$$
\frac{\mathrm{d} \sigma}{\mathrm{d} \hat{k}_{1} \mathrm{~d} \hat{k}_{2} \mathrm{~d} \varepsilon}=(2 \pi)^{4} \frac{k_{1} k_{2}}{k}\left|T_{f i}\right|^{2},
$$

The initial state, which takes into account the incident and the bound electrons, will be written as a product of a plane wave and an exact wave function of the hydrogen atom:

$$
\Psi_{i}^{0}\left(\boldsymbol{r}_{1}, \boldsymbol{r}_{2}\right)=\frac{\mathrm{e}^{i \boldsymbol{k} \cdot r_{2}}}{(2 \pi)^{3 / 2}} \varphi_{i}\left(\boldsymbol{r}_{1}\right),
$$

which eventually is taken in the zero angular momentum state. The final state $\Psi_{f}$ is given by $\Psi_{f} \cong \Psi_{G H F}$ to be evaluated in the SCF approximation. The continuum wave functions are normalized to a $\delta$ function in momentum space, as in (2.3).

As in the Hartree-Fock case, the resulting system of mutually coupled SCF equations is solved by iteration, subject to the self consistency requirement. First one guesses a set of scattering wave functions. Then one computes the SCF potentials and the inhomogeneous terms. The set of Equation (3.4) is then solved. These new scattering wave functions are amputated and used to compute the new SCF potentials and the new inhomogeneous terms, which in turn are used to obtain a third set of scattering wave functions. The cycle continues until self consistency is reached within some preset tolerance. The numerical method we adopt for the scattering functions is the use of the distorted Green's functions. In this method, we transform the set of differential equations that the scattering functions satisfy, along with their corresponding boundary conditions, into integral equations. The final step in the numerical procedure is to calculate the proper integral expressions for the scattering amplitudes $T_{f i}$ and the corresponding ionization cross section.

\section{Results}

We first choose the GHF wave function of the scattering system, as in the case of Hartree-Fock, in its lowest approximation: $\Psi_{G H F}=\Psi_{G H F}^{0}$. We carried out calculations at total energies $E_{t}=2.0 \mathrm{Ry}$ and $E_{t}=3.0 \mathrm{Ry}$, since the previous data are available for these energies for comparison [10] [20]. Our results for the triplet 
Table 1. Single-differential ionization cross sections $\frac{\mathrm{d} \sigma}{\mathrm{d} \varepsilon}$ (in units of $\pi a_{0}^{2} /$ hartree) of e-H scattering system at $E=2 R y$ and $3 R y$ are given as a function of the energy fraction $\frac{\varepsilon}{E}$ for $S=1$ case where $E=E_{1}^{2}+E_{2}^{2}=\varepsilon+(E-\varepsilon)$. The Exterior Complex Scaling is denoted by $E C S$ and the Generalized Hartree-Fock approach is denoted by $G H F$.

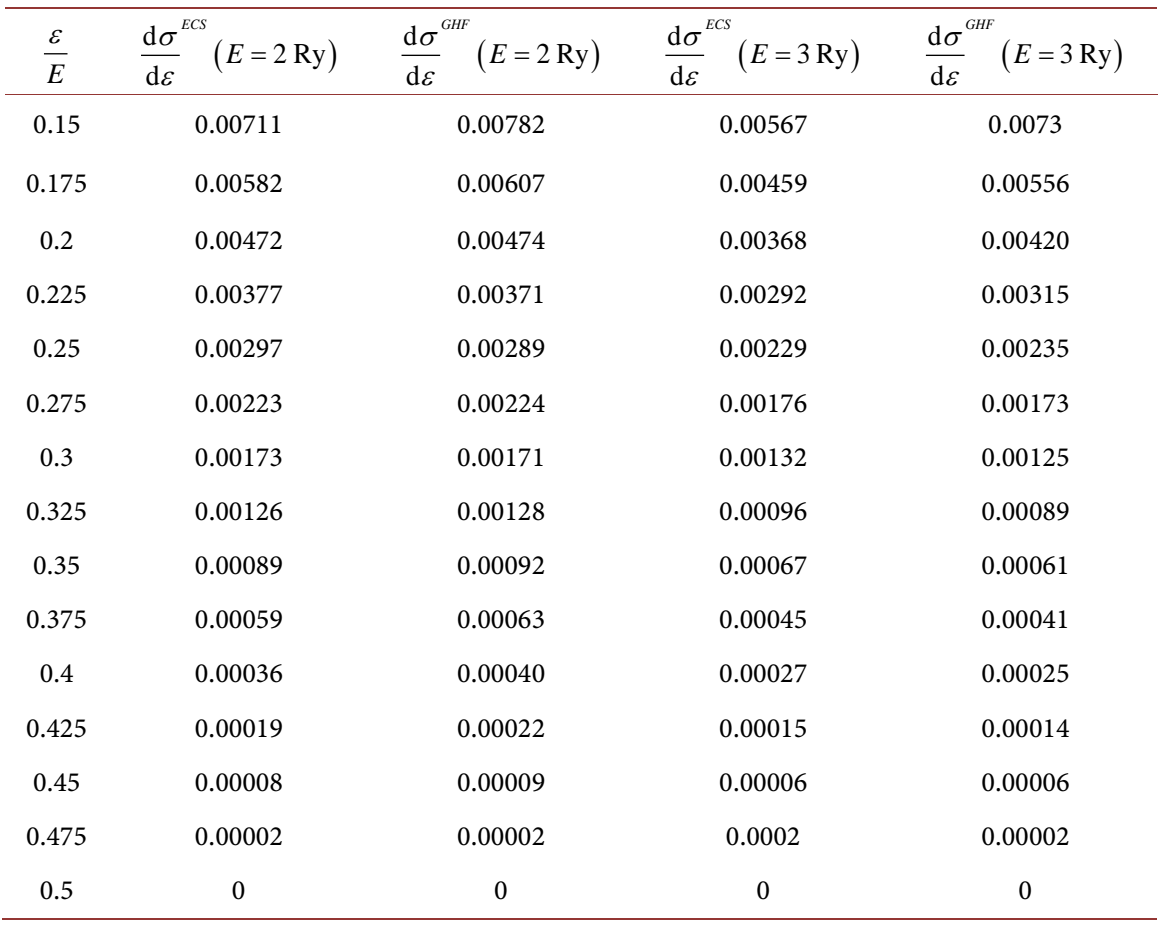

case are reported in Table 1 and compared with the exterior complex scaling [20] (ECS) method. The ECS method seems to be the most accurate procedure available for computing ionization cross section for the model. It uses a two-dimensional grid to solve for the outgoing scattered wave function without explicitly imposing the asymptotic boundary condition for the three charged particles. The coordinates are then scaled by a complex phase factor, beyond a certain radius where the tail of the Coulomb potential is ignored. As a result, the scattered wave function decays like a bound state wave function, which makes the asymptotic boundary condition simple to satisfy. Figure 1 contains additional data obtained by the Convergent Close Coupling (CCC) method [21]. Agreement between our results and all the others is very good for the triplet case. This is especially surprising because the GHF approximation is considered here only in its lowest approximation. Apparently, the amputated wave functions carry much of the essential dynamical information contained in the scattering functions. Hence, we have been able to impose self consistency as a result of the amputation procedure which carefully gets rid of the long range tail represented by the asymptotic part of the scattering functions. Another feature of the GHF approach is that, by definition, our ionization differential cross sections are symmetric about the point $\frac{\varepsilon}{E}=0.5$. This property is absent in the close-coupling approach. 


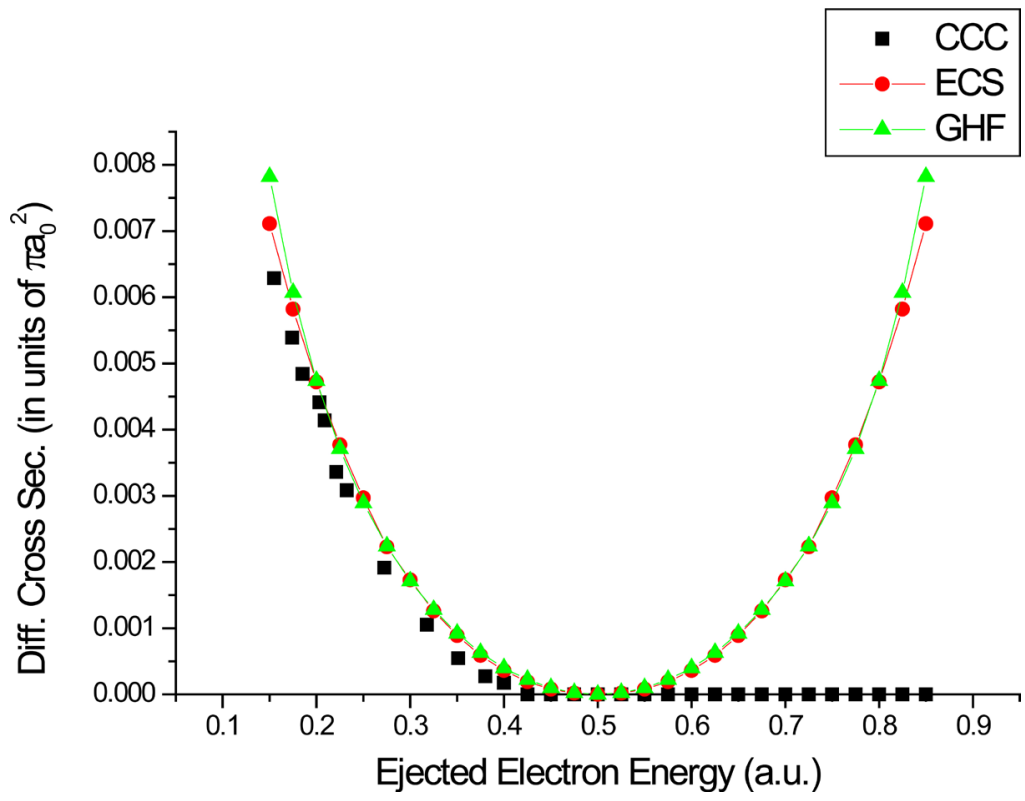

Figure 1. The single-differential ionization cross sections (in units of $\pi a_{0}^{2} /$ hartree) of e-H scattering system at $E=1$ hartree for the $S=1$ case is given as a function of the ejected electron energy. The GHF results are compared with ECS and CCC methods.

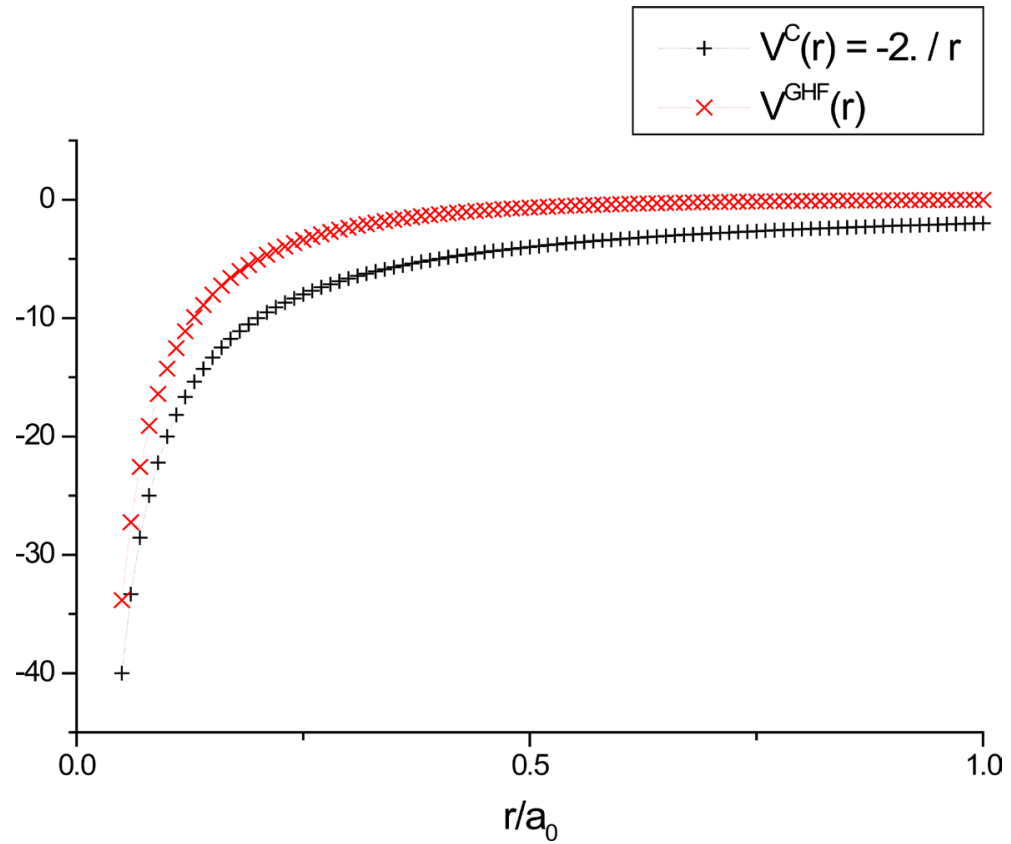

Figure 2. Comparison of the Coulombic potential (cross + ) and the GHF potential (cross x) for the continuum electron for the $S=1$ case at $\frac{\varepsilon}{E}=0.5$.

In Figure 2 we display the interaction potential $V^{\mathrm{GHF}}$ between the two continuum electrons. This is the first time the SCF potential is displayed in the ionization problem. It is then compared to the pure Coulombic potential $V^{\complement}$. Figure 3 shows that the amputated wave function $X$ which carries all the dynamics is of short range. It is presumably due to the character of the present model. 


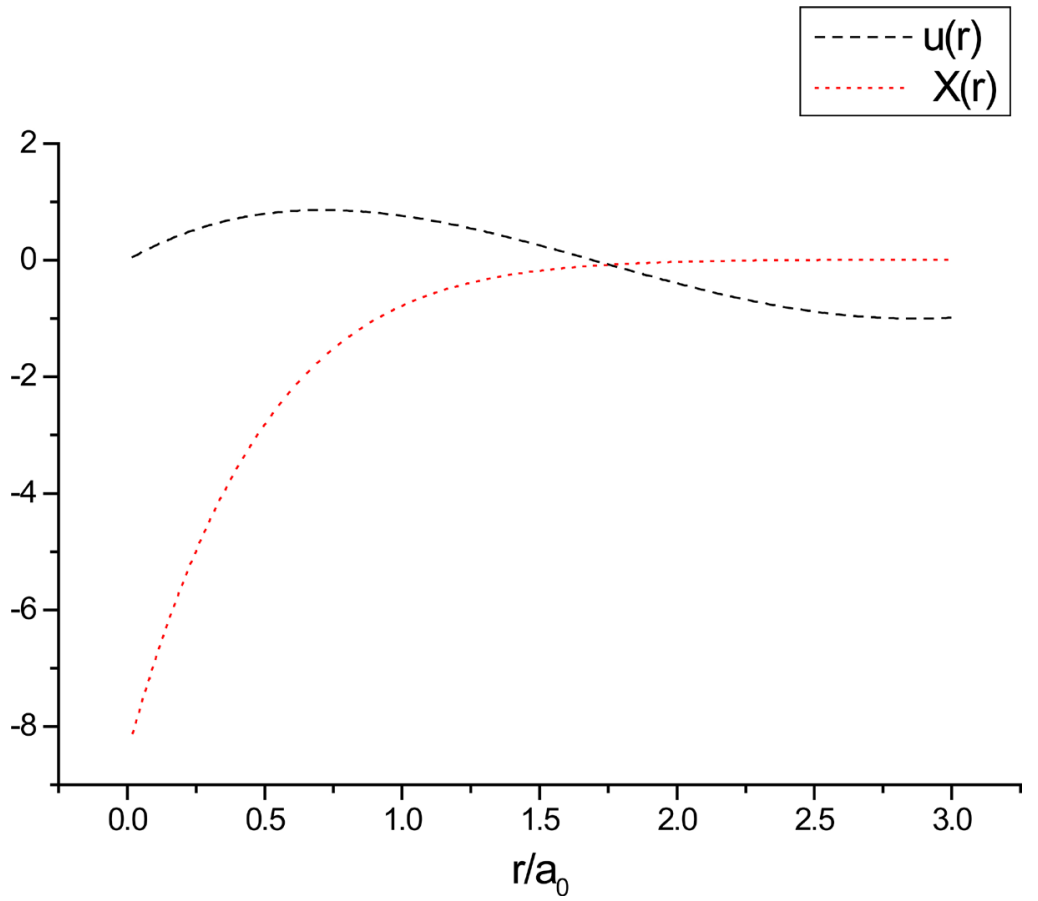

Figure 3. Plot of the continuum wave function $\mathrm{u}$ obtained by the GHF procedure and its corresponding amputated wave function $X$, for the $S=1$ case at $\frac{\varepsilon}{E}=0.5$.

Our results for the singlet case are reported in Table 2. Figure 4 shows that the singlet case is more problematic, as the existing theories are not all in agreement. Since the GHF is quite distinct from all the other approaches and based on the potentially powerful SCF approach, further analyses should help clarify the situation.

\section{Analysis of the GHF Wave Functions}

From the result of the GHF treatment given in the previous section, it is clear that the GHF functions in the single configuration approximation are already in nearly exact form for all values of $\frac{\varepsilon}{E}$ for $S=1$ and for $\frac{\varepsilon}{E}<0.2$ for the $S=0$ state. For these regions of small $\frac{\varepsilon}{E}$, no configuration mixing seems necessary. This is rather surprising, because the ionization process is presumably a more complicated process as compared to the other elastic and inelastic collisions. Although it was shown [16] formally that a "proper" projection of the scattering equation can yield the "exact" amplitude, it is in general difficult to construct such a projection. Apparently, the projections employed in the GHF in terms of the amputated scattering functions seem to be very effective.

Since we have nearly "exact" wave functions for low $\frac{\varepsilon}{E}$, it is of interest to examine the wave functions generated by other approximation procedures, such as the plane waves, pure Coulomb waves, and the effective charge Coulomb 
Table 2. Single-differential ionization cross sections $\frac{\mathrm{d} \sigma}{\mathrm{d} \varepsilon}$ (in units of $\pi a_{0}^{2} /$ hartree) of e-H scattering system at $E=2 R y$ and $3 R y$ are given as a function of the energy fraction $\frac{\varepsilon}{E}$ for the $S=0$ case. The Exterior Complex Scaling is denoted by ECS and the Generalized Hartree-Fock approach is denoted by GHF.v.

\begin{tabular}{ccccc}
\hline$\frac{\varepsilon}{E}$ & $\frac{\mathrm{d} \sigma^{E C S}}{\mathrm{~d} \varepsilon}(E=2 \mathrm{Ry})$ & $\frac{\mathrm{d} \sigma^{\text {GHF }}}{\mathrm{d} \varepsilon}(E=2 \mathrm{Ry})$ & $\frac{\mathrm{d} \sigma^{E C S}}{\mathrm{~d} \varepsilon}(E=3 \mathrm{Ry})$ & $\frac{\mathrm{d} \sigma^{G H F}}{\mathrm{~d} \varepsilon}(E=3 \mathrm{Ry})$ \\
\hline 0.15 & 0.04542 & 0.04263 & 0.02278 & 0.02648 \\
0.175 & 0.04387 & 0.03903 & 0.0218 & 0.02362 \\
0.2 & 0.04242 & 0.03527 & 0.0209 & 0.02076 \\
0.225 & 0.04103 & 0.03174 & 0.02008 & 0.0179 \\
0.25 & 0.03969 & 0.02861 & 0.01932 & 0.01504 \\
0.275 & 0.03838 & 0.02587 & 0.01861 & 0.01361 \\
0.3 & 0.03709 & 0.02348 & 0.01793 & 0.01215 \\
0.325 & 0.03581 & 0.02137 & 0.01728 & 0.01085 \\
0.35 & 0.03453 & 0.01949 & 0.01665 & 0.00969 \\
0.375 & 0.03323 & 0.01788 & 0.01603 & 0.00866 \\
0.4 & 0.03192 & 0.01656 & 0.01543 & 0.00775 \\
0.425 & 0.03063 & 0.01545 & 0.01484 & 0.00694 \\
0.45 & 0.0294 & 0.01448 & 0.01428 & 0.00622 \\
0.475 & 0.02838 & 0.01367 & 0.01383 & 0.00559 \\
0.5 & 0.02792 & 0.01307 & 0.01362 & 0.00506 \\
\hline
\end{tabular}

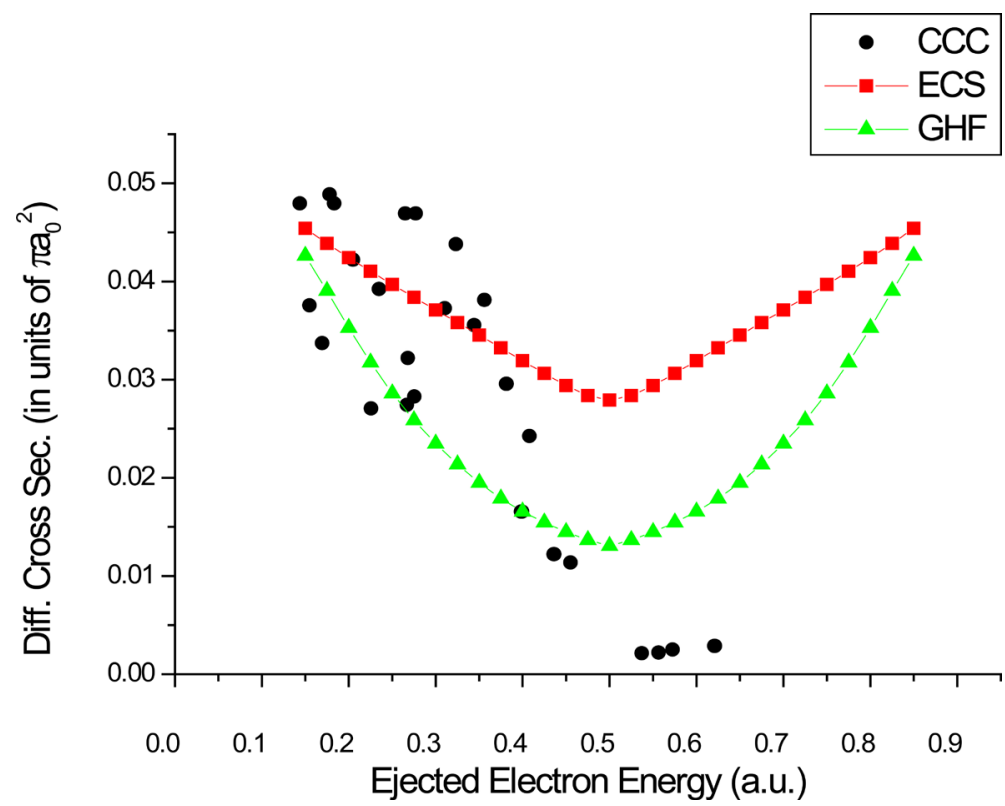

Figure 4. The single-differential ionization cross sections (in units of $\pi a_{0}^{2} /$ hartree) of e-H scattering system at $E=1$ hartree for the $S=0$ case is given as a function of the ejected electron energy. The GHF results are compared with ECS and CCC methods. 
waves. In particular, the effective charge approximation can be critically analyzed. In the conventional approach to ionization, the integrals for the "post" form of the ionization amplitude do not converge unless the so-called Peterkop condition is met. That is, for the two effective charges $Z_{1}$ and $Z_{2}$, it is required that

$$
Z_{1} / k_{1}+Z_{2} / k_{2}=1 / k_{1}+1 / k_{2}-1 /\left|\boldsymbol{k}_{1}-\boldsymbol{k}_{2}\right| .
$$

Thus, in the limit of $k_{1} \rightarrow 0$, we have $Z_{1} \rightarrow 1$. Table 3 and Table 4 illustrate the wave functions in the various approximations, as they are compared with the GHF functions. The effective charges used in the tables are adjusted to simulate

Table 3. Wave functions generated for the $S=0$ case with $Z_{1}=0.1, Z_{2}=0.2$ at $\frac{\varepsilon}{E}=0.4$ : $\mathrm{B}=$ plane waves, $\mathrm{P}=$ pure Coulomb waves, $\mathrm{Z}=$ effective charge Coulomb waves, and G:GHF.

\begin{tabular}{|c|c|c|c|c|c|c|c|c|}
\hline$r$ & $u_{1 \mathrm{~B}}(r)$ & $u_{1 \mathrm{P}}(r)$ & $u_{1 \mathrm{ZZ}}(r)$ & $u_{1 \mathrm{G}}(r)$ & $u_{2 \mathrm{~B}}(r)$ & $u_{2 \mathrm{P}}(r)$ & $u_{2 \mathrm{ZZ}}(r)$ & $u_{2 \mathrm{G}}(r)$ \\
\hline 0.2 & 0.178 & 0.383 & 0.206 & 0.106 & 0.217 & 0.424 & 0.271 & 0.578 \\
\hline 0.4 & 0.35 & 0.601 & 0.397 & 0.251 & 0.424 & 0.658 & 0.507 & 0.841 \\
\hline 0.6 & 0.511 & 0.68 & 0.567 & 0.412 & 0.611 & 0.729 & 0.699 & 0.957 \\
\hline 0.8 & 0.656 & 0.646 & 0.712 & 0.567 & 0.768 & 0.669 & 0.838 & 1 \\
\hline 1 & 0.78 & 0.527 & 0.827 & 0.705 & 0.889 & 0.509 & 0.921 & 0.995 \\
\hline 1.2 & 0.879 & 0.348 & 0.909 & 0.82 & 0.967 & 0.285 & 0.946 & 0.942 \\
\hline 1.4 & 0.95 & 0.136 & 0.956 & 0.909 & 0.999 & 0.027 & 0.912 & 0.846 \\
\hline 1.6 & 0.99 & -0.089 & 0.967 & 0.969 & 0.983 & -0.234 & 0.825 & 0.709 \\
\hline 1.8 & 0.999 & -0.308 & 0.942 & 0.997 & 0.921 & -0.472 & 0.69 & 0.538 \\
\hline 2 & 0.976 & -0.503 & 0.883 & 0.994 & 0.814 & -0.667 & 0.516 & 0.342 \\
\hline 2.2 & 0.922 & -0.662 & 0.793 & 0.959 & 0.668 & -0.804 & 0.313 & 0.13 \\
\hline 2.4 & 0.839 & -0.776 & 0.674 & 0.894 & 0.49 & -0.874 & 0.0924 & -0.089 \\
\hline 2.6 & 0.728 & -0.839 & 0.531 & 0.8 & 0.289 & -0.873 & -0.133 & -0.303 \\
\hline 2.8 & 0.595 & -0.851 & 0.37 & 0.681 & 0.0743 & -0.803 & -0.351 & -0.503 \\
\hline 3 & 0.442 & -0.811 & 0.196 & 0.539 & -0.144 & -0.673 & -0.55 & -0.679 \\
\hline 3.2 & 0.276 & -0.723 & 0.0152 & 0.381 & -0.356 & -0.492 & -0.72 & -0.822 \\
\hline 3.4 & 0.1 & -0.595 & -0.166 & 0.21 & -0.55 & -0.276 & -0.853 & -0.926 \\
\hline 3.6 & -0.0783 & -0.433 & -0.342 & 0.033 & -0.719 & -0.04 & -0.94 & -0.986 \\
\hline 3.8 & -0.254 & -0.249 & -0.506 & -0.145 & -0.853 & 0.199 & -0.978 & -0.999 \\
\hline 4 & -0.422 & -0.051 & -0.652 & -0.319 & -0.946 & 0.424 & -0.966 & -0.964 \\
\hline 4.2 & -0.577 & 0.15 & -0.777 & -0.483 & -0.994 & 0.621 & -0.903 & -0.882 \\
\hline 4.4 & -0.713 & 0.343 & -0.876 & -0.631 & -0.994 & 0.776 & -0.794 & -0.759 \\
\hline 4.6 & -0.826 & 0.519 & -0.944 & -0.759 & -0.947 & 0.88 & -0.644 & -0.599 \\
\hline 4.8 & -0.913 & 0.67 & -0.981 & -0.863 & -0.855 & 0.927 & -0.461 & -0.411 \\
\hline 5 & -0.971 & 0.787 & -0.986 & -0.939 & -0.721 & 0.914 & -0.255 & -0.203 \\
\hline
\end{tabular}


Table 4. Wave functions generated for the $S=0$ case with $Z_{1}=0.05, Z_{2}=0.4$ at $\frac{\varepsilon}{E}=0.1$ : $\mathrm{B}=$ plane waves, $\mathrm{P}=$ pure Coulomb waves, $\mathrm{Z}=$ effective charge Coulomb waves, and $\mathrm{G}: \mathrm{GHF}$

\begin{tabular}{|c|c|c|c|c|c|c|c|c|}
\hline$r$ & $u_{1 \mathrm{~B}}(r)$ & $u_{1 \mathrm{P}}(r)$ & $u_{1 Z 1}(r)$ & $u_{1 \mathrm{G}}(r)$ & $u_{2 \mathrm{~B}}(r)$ & $u_{2 \mathrm{P}}(r)$ & $u_{2 \mathrm{ZZ}}(r)$ & $u_{2 \mathrm{G}}(r)$ \\
\hline 0.2 & 0.0893 & 0.272 & 0.104 & 0.0555 & 0.265 & 0.468 & 0.364 & 0.609 \\
\hline 0.4 & 0.178 & 0.433 & 0.206 & 0.132 & 0.511 & 0.716 & 0.643 & 0.874 \\
\hline 0.6 & 0.265 & 0.504 & 0.303 & 0.218 & 0.721 & 0.771 & 0.826 & 0.981 \\
\hline 0.8 & 0.35 & 0.503 & 0.397 & 0.305 & 0.879 & 0.667 & 0.906 & 1 \\
\hline 1 & 0.432 & 0.448 & 0.485 & 0.389 & 0.974 & 0.45 & 0.885 & 0.951 \\
\hline 1.2 & 0.511 & 0.353 & 0.567 & 0.47 & 0.999 & 0.163 & 0.772 & 0.834 \\
\hline 1.4 & 0.586 & 0.232 & 0.643 & 0.547 & 0.953 & -0.146 & 0.584 & 0.657 \\
\hline 1.6 & 0.656 & 0.0954 & 0.712 & 0.619 & 0.839 & -0.437 & 0.341 & 0.434 \\
\hline 1.8 & 0.721 & -0.047 & 0.773 & 0.687 & 0.664 & -0.675 & 0.0659 & 0.179 \\
\hline 2 & 0.78 & -0.187 & 0.827 & 0.749 & 0.442 & -0.836 & -0.215 & -0.088 \\
\hline 2.2 & 0.833 & -0.318 & 0.872 & 0.805 & 0.189 & -0.904 & -0.477 & -0.349 \\
\hline 2.4 & 0.879 & -0.435 & 0.909 & 0.855 & -0.078 & -0.875 & -0.698 & -0.585 \\
\hline 2.6 & 0.918 & -0.534 & 0.937 & 0.898 & -0.34 & -0.754 & -0.86 & -0.779 \\
\hline 2.8 & 0.95 & -0.612 & 0.956 & 0.934 & -0.577 & -0.557 & -0.95 & -0.917 \\
\hline 3 & 0.974 & -0.669 & 0.966 & 0.962 & -0.773 & -0.304 & -0.961 & -0.99 \\
\hline 3.2 & 0.99 & -0.702 & 0.967 & 0.982 & -0.913 & -0.021 & -0.893 & -0.992 \\
\hline 3.4 & 0.999 & -0.712 & 0.959 & 0.995 & -0.989 & 0.264 & -0.753 & -0.923 \\
\hline 3.6 & 0.999 & -0.699 & 0.942 & 1 & -0.993 & 0.524 & -0.552 & -0.788 \\
\hline 3.8 & 0.992 & -0.666 & 0.917 & 0.997 & -0.926 & 0.735 & -0.306 & -0.596 \\
\hline 4 & 0.976 & -0.613 & 0.883 & 0.986 & -0.794 & 0.879 & -0.036 & -0.362 \\
\hline 4.2 & 0.953 & -0.543 & 0.842 & 0.966 & -0.604 & 0.942 & 0.237 & -0.102 \\
\hline 4.4 & 0.922 & -0.459 & 0.793 & 0.94 & -0.371 & 0.919 & 0.491 & 0.165 \\
\hline 4.6 & 0.884 & -0.362 & 0.736 & 0.905 & -0.111 & 0.815 & 0.707 & 0.421 \\
\hline 4.8 & 0.839 & -0.256 & 0.674 & 0.864 & 0.156 & 0.638 & 0.867 & 0.646 \\
\hline 5 & 0.787 & -0.144 & 0.605 & 0.815 & 0.412 & 0.405 & 0.959 & 0.826 \\
\hline
\end{tabular}

the GHF functions; for $\frac{\varepsilon}{E}=0.1$, we have determined that approximately $Z_{1} \approx$ 0.05 and $Z_{2} \approx 0.4$, while for $\frac{\varepsilon}{E}=0.4, Z_{1} \approx 0.1$ and $Z_{2} \approx 0.2$. Evidently, the Peterkop condition breaks down in the present model. In fact, such a condition was originally proposed for mathematical consistency and not for dynamical reasons. The "prior" form of the amplitude does not have the convergence difficulty and thus such a condition is not necessary. The pure Coulombic functions with $Z=1$ also totally fail.

Another surprising result of the GHF treatment is that the $k_{1}$ component $\left(<k_{2}\right)$ 
of the GHF wave function turned out to be nearly identical to the plane wave, in spite of complicated scattering equations they satisfy, including the full exchange terms. This is contrary to the conventional physical picture of more distortion for lower energy scattering. Apparently, several components in the equation work to cancel each other's effects. It also strongly suggests that much of the earlier calculations all agree at small $\varepsilon$ because the low energy component of the final wave functions are more or less that of free particle. Further, the higher energy component with $k_{2}$ is only partially distorted by the coulomb potential, with the effective charge of roughly $Z_{2} \approx 0.4$.

The triplet case is also of some interest, as all the calculations more or less agree with each other for all $\frac{\varepsilon}{E}$. The reason for this may be that the scattering functions for the triplet state have a node at $r_{1}=r_{2}$, which places a stringent constraint on the wave functions. However, the magnitude of the wave functions at the peaks near $r_{2} \approx r_{1} / 2$ can still be quite different among the various theories. In Figures 5-8, we plotted the wave functions in the various approximations, at several sample points of $r_{1}\left(>r_{2}\right)$. It clearly shows that the various wave functions are all similar in the triplet case, but not for the singlet.

Eventually, configuration mixing is needed to improve the wave functions in the singlet case with $\frac{\varepsilon}{E}>0.2$. Such mixing was carried out previously in the GHF context for the electron-hydrogen scattering system, by adding new channels and solving a set of coupled equations. Alternatively, a variational treatment may also be possible, as in the bound state case.

A part of our study in progress includes an extension of additional channels in the total wave function to test the convergence of the theory. We adopt a vartiational procedure to improve on the ionization differential cross section. This

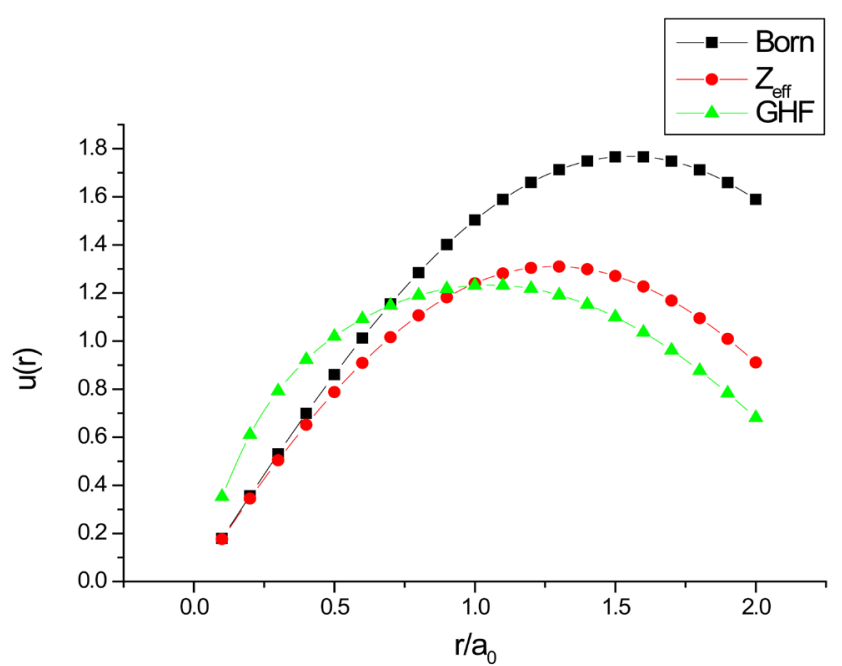

(a)

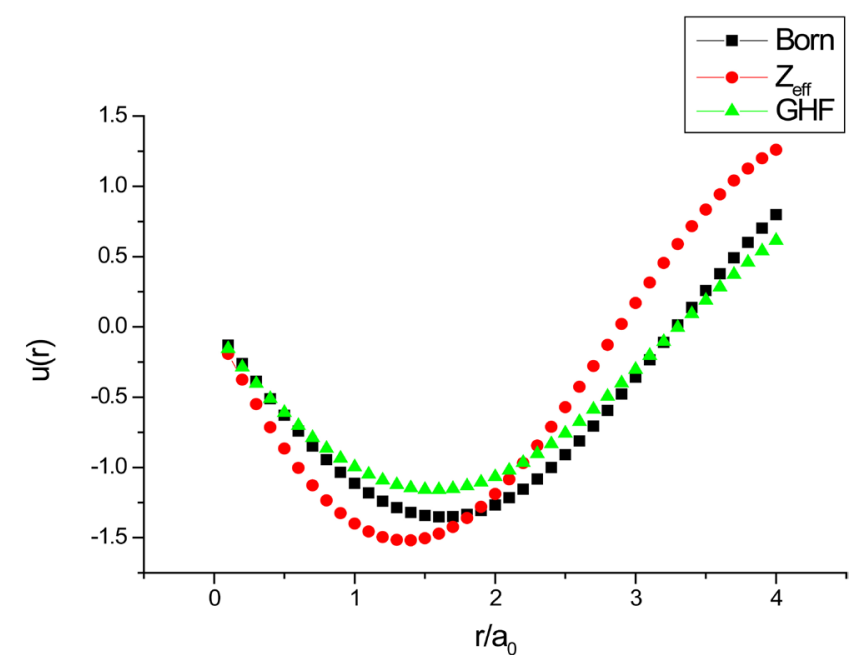

(b)

Figure 5. Comparison of the plane wave functions, Coulombic wave functions with effective charges $\left(Z_{1}=0.1, Z_{2}=0.2\right)$, and GHF wave functions the $S=0$ case at $\frac{\varepsilon}{E}=0.4$. 


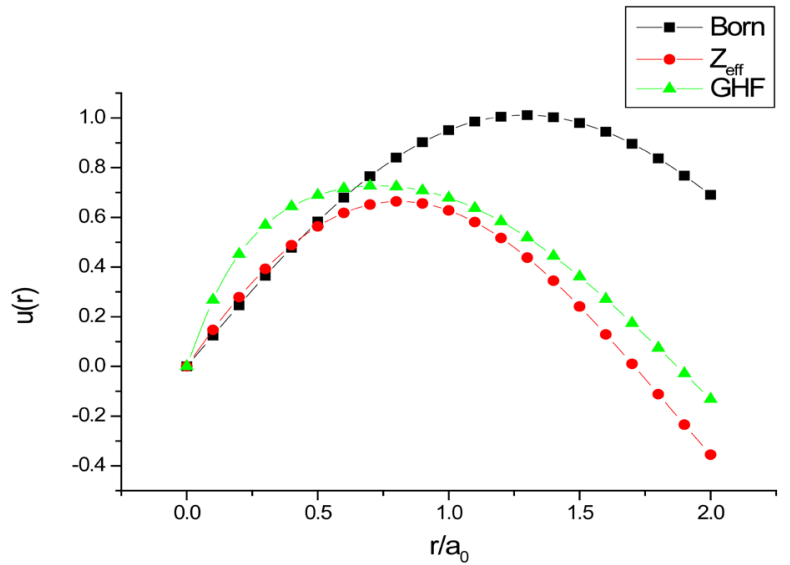

(a)

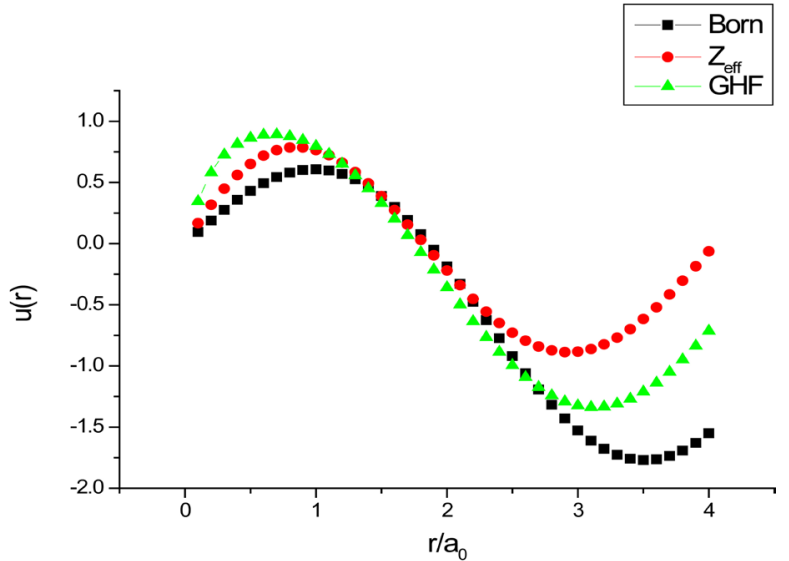

(b)

Figure 6. Comparison of the plane wave functions, Coulombic wave functions with effective charges $\left(Z_{1}=0.05, Z_{2}=0.4\right)$, and GHF wave functions the $S=0$ case at $\frac{\varepsilon}{E}=0.1$.

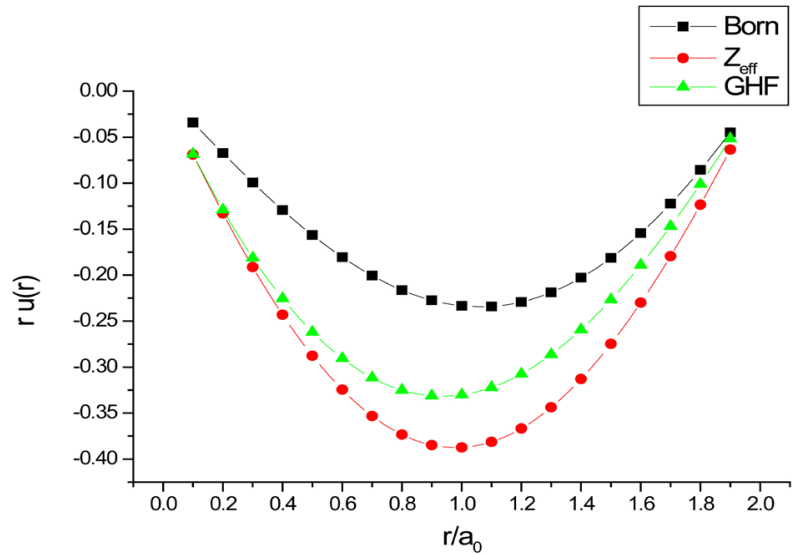

(a)

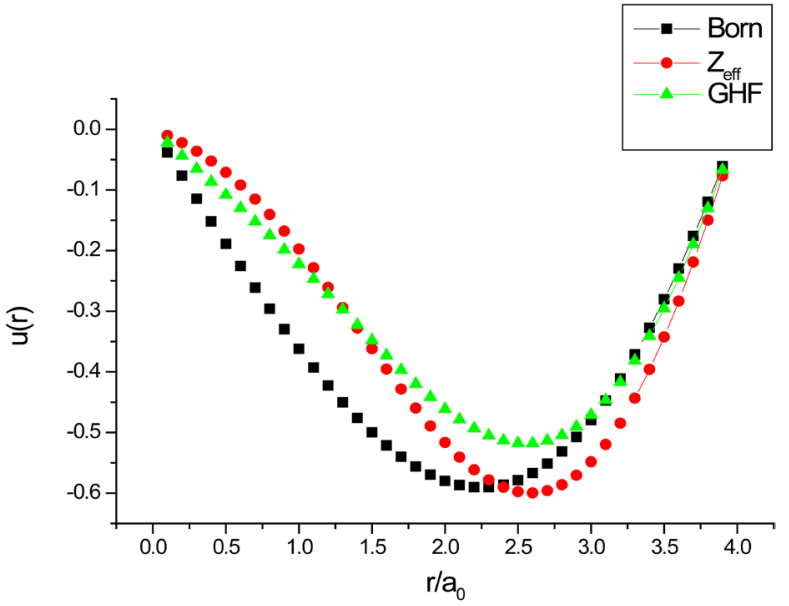

(b)

Figure 7. Comparison of the plane wave functions, Coulombic wave functions with effective charges $\left(Z_{1}=0.1, Z_{2}=0.2\right)$, and GHF wave functions the $S=1$ case at $\frac{\varepsilon}{E}=0.4$.

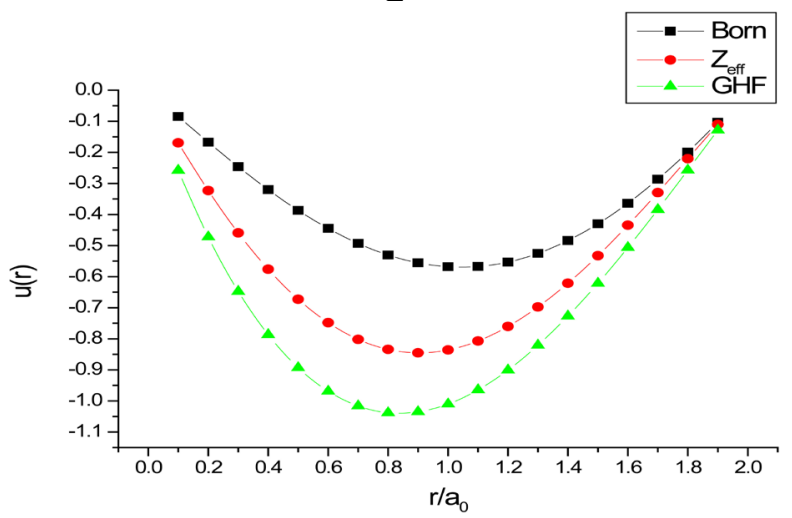

(a)

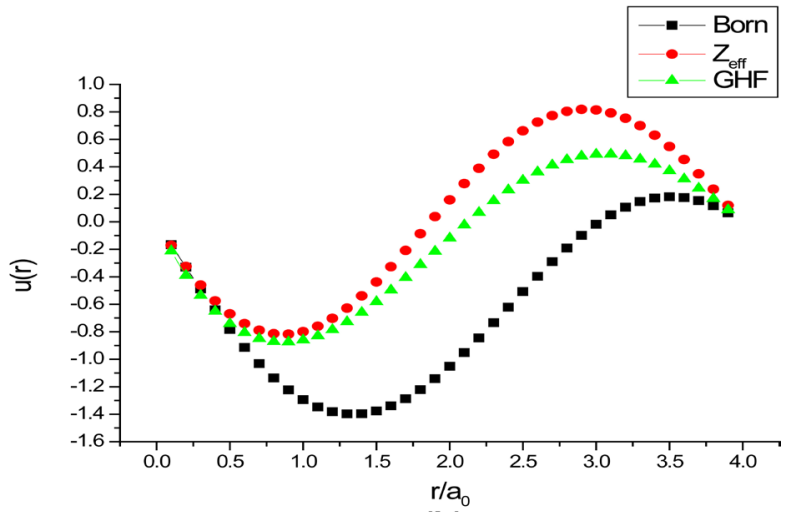

(b)

Figure 8. Comparison of the plane wave functions, Coulombic wave functions with effective charges $\left(Z_{1}=0.05, Z_{2}=0.4\right)$, and GHF wave functions the $S=1$ case at $\frac{\varepsilon}{E}=0.1$. 
work will be reported elsewhere.

\section{Conclusion}

The present study shows that the GHF approach is a viable method to treat complex collision systems. The effectiveness of the theory may be attributed to the crucial physics information carried by the amputated wave function. As emphasized in [16], it is possible in principle to obtain accurate solutions to the collision problem by adopting a "proper projection" of the scattering equations, although it is in general difficult to find the proper projection. However, the GHF seems to provide a partial answer to the projection problem, in terms of the amputated functions $X$. For application of GHF to more realistic and complex systems, it is important to better understand the role played by the $X$ s. The multiconfiguration extension of the GHF is in progress to clarify the situation.

\section{Acknowledgements}

We acknowledge the Optical Science Center for Applied Research (OSCAR) and the financial support of the National Science Foundation (NSF-CREST grant \# 1242067).

\section{References}

[1] Bray, I. and Stelbovics, A. (1992) Physical Review Letters, 69, 53.

[2] Callaway, J. and Oza, D.H. (1984) Physical Review D, 29, 2416. https://doi.org/10.1103/PhysRevD.29.567

[3] Kato, D. and Watanbe, S. (1995) Physical Review Letters, 74, 2443. https://doi.org/10.1103/PhysRevLett.74.2443

[4] Meyer, K.W., Greene, C.H. and Bray, I. (1995) Physical Review A, 52, 13344. https://doi.org/10.1103/PhysRevA.52.1334

[5] Ihra, W., Draeger, M., Handke, G. and Friedrich, H. (1995) Physical Review A, 52, 3752. https://doi.org/10.1103/PhysRevA.52.3752

[6] Pindzola, M.S. and Schultz, D.R. (1996) Physical Review A, 53, 1525. https://doi.org/10.1103/PhysRevA.53.1525

[7] Bartschat, K. and Bray, I. (1996) Physical Review A, 54, R1002. https://doi.org/10.1103/PhysRevA.54.R1002

[8] Childers, J.G., James, K.E., Hughes, M., Bray, I., Baertschy, M. and Khakoo, M.A. (2003) Physical Review A, 68, 030702. https://doi.org/10.1103/PhysRevA.68.030702

[9] Jones, S. and Madison, D.H. (2003) Physical Review Letters, 91, 073201. https://doi.org/10.1103/PhysRevLett.91.073201

[10] Baertschy, M., Rescigno, T.N., Isaacs, W.A. and McCurdy, C.W. (1999) Physical Review A, 60, R13. https://doi.org/10.1103/PhysRevA.60.R13

[11] Bray, I. and Stelbovics, A. (1993) Physical Review Letters, 70, 746.

[12] Hartree, D.R. (1928) Proceedings of the Cambridge Philosophical Society, 24, 426. https://doi.org/10.1017/S0305004100015954

[13] Fock, V. (1930) Journal of Physics, 61, 126.

[14] Dirac, P.A.M. (1930) Proceedings of the Cambridge Philosophical Society, 26, 361. 
https://doi.org/10.1017/S0305004100016091

[15] Fetter, A.L. and Walecka, D. (1971) Quantum Theory of Many-Particle Systems. McGraw-Hill, New York.

[16] Hahn, Y. (1996) Journal of Physics B, 29, 1385.

Hahn, Y. (1996) Journal of Physics A, 29, 7301.

[17] Hahn, Y. (1997) Physical Review A, 55, 4238.

https://doi.org/10.1103/PhysRevA.55.4238

[18] Zerrad, E. and Hahn, Y. (1999) Physics Letters A, 254, 341.

[19] Zerrad, E. and Hahn, Y. (2000) The European Physical Journal D, 10, 81-97. https://doi.org/10.1007/s100530050528

[20] Jones, S. and Stelbovics, A.T. (2000) Physical Review Letters, 84, 1878. https://doi.org/10.1103/PhysRevLett.84.1878

[21] Bray, I. (1997) Physical Review Letters, 78, 4721. https://doi.org/10.1103/PhysRevLett.78.4721

Submit or recommend next manuscript to SCIRP and we will provide best service for you:

Accepting pre-submission inquiries through Email, Facebook, LinkedIn, Twitter, etc. A wide selection of journals (inclusive of 9 subjects, more than 200 journals) Providing 24-hour high-quality service User-friendly online submission system Fair and swift peer-review system Efficient typesetting and proofreading procedure Display of the result of downloads and visits, as well as the number of cited articles Maximum dissemination of your research work

Submit your manuscript at: http://papersubmission.scirp.org/ Or contact jmp@scirp.org 